\title{
PHYTOCHEMICAL STUDY OF BIOACTIVE CONSTITUENTS FROM SATUREJA MONTANA L. GROWING IN EGYPT AND THEIR ANTIMICROBIAL AND ANTIOXIDANT ACTIVITIES
}

\author{
ALI M EL-HAGRASSI ${ }^{1 *}$, WALID E ABDALLAH ${ }^{2}$, ABEER F OSMAN ${ }^{3}$, KHALED A ABDELSHAFEEK ${ }^{2,4}$ \\ ${ }^{1}$ Department of Phytochemistry and Plant Systematic, Pharmaceutical Industries Div., National Research National Research Centre, \\ 33 El Bohouth St.(former El Tahrir St.), Dokki, Giza, Egypt, P.o. 12622, ${ }^{2}$ Department of Phytochemistry, Pharmaceutical Industries Div., \\ National Research Centre, 33 El Bohouth St.(former El Tahrir St.), Dokki, Giza, Egypt, P.o.12622. ${ }^{3}$ Department of Chemistry of Natural \\ Compounds, Pharmaceutical Industries Div., National Research Centre, 33 El Bohouth St.(former El Tahrir St.), Dokki, Giza, Egypt, \\ P.o.12622. ${ }^{4}$ Department of Chemistry, Faculty of Science, El-Baha University, KSA. Email: alielhagrasi@gmail.com
}

Received: 25 September 2017, Revised and Accepted: 26 December 2017

ABSTRACT

Objective: This work aimed to investigate the lipid constituents and flavonoidal compounds of Satureja montana, in addition to evaluation of different extracts and/or isolated compounds as antimicrobials and antioxidants.

Methods: The volatile and lipid constituents were extracted with n-hexane by partition from hydroalcoholic extract of $S$. montana L. aerial parts, after then were fractionated to unsaponifiable matters and fatty acid methyl esters which were identified by gas-liquid chromatography and/or gas chromatography-mass spectrometry. The phenolic constituents were isolated from the ethyl acetate fraction of the aqueous methanolic extract of the aerial parts of the plant. The antimicrobial activity of different extracts and the isolated compounds was evaluated against Gram-positive, Gramnegative bacteria, yeast, and fungus using a modified Kirby-Bauer disc diffusion method.

Results: The identified compounds are luteolin-7-rhamnoside-4'-O- $\beta$-glucopyranoside (1), quercetin-3-O- $\alpha$-L-rhamnopyranoside (2), quercetin-7-Oglucopyranoside (3), luteolin-7-O-glucopyranoside (4), 5-hydroxy-6,7,8,4'-tetramethoxy flavone (5), gallic acid (6), 2,3-hexahydroxydiphenoyl 1-galloyl glucopyranoside (7), and quercetin (8). The structure of all isolated compounds was established using different chromatographic and spectroscopic measurements (PC, thin-layer chromatography, ultraviolet [UV], 1D, 2D-nuclear magnetic resonance, and MS). Compound-2 showed the highest antibacterial activity against all the tested microorganisms. Hydroalcoholic extract exhibited high antioxidant activity (87.7\%). On the other hand, hexane fraction showed a low antioxidant activity (46.4\%), in addition to the compound-8 showed the highest antioxidant activity (96.27\%) in 2,2-diphenyl-1-picrylhydrazyl assay.

Conclusion: It can be concluded that the hydroalcoholic extract of S. montana showed significant antimicrobial and antioxidant activity.

Keywords: Satureja montana, Family Lamiaceae, Lipid constituents, Flavonoids, Antimicrobial, Antioxidant activity.

(c) 2018 The Authors. Published by Innovare Academic Sciences Pvt Ltd. This is an open access article under the CC BY license (http://creativecommons. org/licenses/by/4. 0/) DOI: http://dx.doi.org/10.22159/ajpcr.2018.v11i4.22756

\section{INTRODUCTION}

The genus Satureja L. belonging to the family Lamiaceae contains about 200 species of aromatic herbs and shrubs that are native to warm temperate regions and may be annual or perennial grow in the Middle East, Mediterranean region to Europe, West Asia, and North Africa. Over 30 species of this genus are distributed in eastern parts of the Mediterranean area [1]. Many of these species have different biological activities such as antibacterial, antifungal, antioxidant, cytotoxic, insecticidal, antidiabetic, anti-leishmanial, insect repellant, hepatoprotective, antiviral, anticholinesterase, hypolipidemic-hypoglycemic, anti-inflammatory, antinociceptive, nematicidal, anti-proliferative, genotoxic, anti-genotoxic, neuroprotective, ovicidal, anti-biofilm, molluscicidal, antihelmintic, herbicidal, anti-epilepsy, anti-Alzheimer, amebicidal, nephroprotective, anti-lipase, wound healing, trypanocidal/anti-protozoal, enzyme inhibition, anti-spasmodic, vasodilatory-vasorelaxant, anti-tumoral, and diuretic activities [2-9]. Previously, phytochemical analysis of Satureja species revealed the presence of volatile oils, phenolic acids, anthocyanins, flavones, diterpenes, triterpenes, and sterols [10-14].

The major constituents of hydrodistilled volatile oils from the aerial parts of Satureja montana and Saxifraga cuneifolia growing in Croatia were identified by gas chromatography-mass spectrometry (GC-MS) and found carvacrol $(17.7 \%)$ and spathulenol $(13.2 \%)$ as the major compounds [15]. Twenty one compounds in the oil of Satureja montana essential oil were identified by GC-FID analyses and carvacrol was the main (60\%) [9]. Many flavonoids were isolated from Satureja khuzistanica [16]. The identified flavonoids are aromadendrin, taxifolin, naringenin, 5,7,3', $5^{\prime}$-tetrahydroxy flavanone, xanthomicrol, acacetin, cirsimaritin, 7-methoxyluteolin, apigenin, cirsilineol, diosmetin, and 6-hydroxyluteolin 7,3'- dimethyl ether, in addition to a new monoterpene-flavonoid known as saturejin, while MatloubiMoghaddam et al., in 2007, isolated $\beta$-sitosterol, $\beta$-sitosterol-3-O- $\beta$ D-glucoside, ursolic acid, and 4,5,6-trihydroxy-3,7-dimethoxyflavone from the dichloromethane extract of the same plant.

Ahamad et al., in 2005 [17], isolated thymol, oleanolic acid, ursolic acid, and caryophyllene oxide from the aerial parts of S. macrantha and these compounds were effective against Artemia salina larvae. While luteolin, oleanolic acid, $\beta$-sitosterol, and diosmetin were isolated from the ethyl acetate and methanol extracts of Satureja sahendica [13]

The surface flavonoids in Satureja thymbra and Satureja spinosa were studied [18] and identified the following compounds: Naringenin, aromadendrin, eriodictyol, taxifolin, apigenin, genkwanin, ladanein, cirsimaritin, thymusin, xanthomicrol, luteolin7-methyl ether, 6-hydroxyluteolin 7,3'-dimethyl ether, 6-hydroxyluteolin 7,3',4'-trimethyl ether, cirsilineol, thymonin, and 8-methoxycirsilineol. 


\section{METHODS}

\section{Plant material}

S. montana L. was cultivated and grown in the farm of National Research Center (NRC), Giza, Egypt, and it was collected in May 2015. The authentication of plant sample was achieved by Dr. Mohammed Algebaly a taxonomist at NRC. A voucher specimen was deposited in the herbarium of the NRC. The aerial parts were air-dried for 2 weeks under laboratory conditions at $28 \pm 2^{\circ} \mathrm{C}$. The dried material was ground using a domestic blender to fine powder.

\section{Instruments and chemicals}

UV spectra were recorded on Shimadzu model UV-240 and 2401 PC spectrophotometer (Shimadzu Inc., Tokyo, Japan). Buchi apparatus was used to determine the melting points of the isolated pure compounds in open capillaries. Nuclear magnetic resonance (NMR) experiments were recorded on Bruker spectrometer (Switzerland) $600\left({ }^{1} \mathrm{H}\right.$ NMR spectra: $600 \mathrm{MHz} ;{ }^{13} \mathrm{C}$ NMR spectra: $150 \mathrm{MHz}$ ). The chemical shifts are given in $\delta(\mathrm{ppm})$ relative to tetramethylsilane $\left(\mathrm{Me}_{4} \mathrm{Si}\right)$. Column chromatography (CC) was carried out on Polyamide S6: (Riedel-De-Haen AG, Seelze Haen AG, Seelze Hanver, Germany) and Sephadex LH-20 (Pharmacia, Uppsala, Sweden). Paper chromatography (PC, descending) Whatman No. 1 and $3 \mathrm{~mm}$ papers, using solvent systems $(1) \mathrm{H}_{2} \mathrm{O}$, (2) $15 \% \mathrm{HOAc}\left(\mathrm{H}_{2} \mathrm{O}\right.$ : $\mathrm{HOAc}$, 85:15), (3) BAW (n-BuOH: HOAc: $\mathrm{H}_{2} \mathrm{O}, 4: 1: 5$, upper layer), and (4) BBPW ( $\mathrm{C}_{6} \mathrm{H}_{6}: \mathrm{n}$-BuOH: Pyridine: $\mathrm{H}_{2} \mathrm{O}, 1: 5: 3: 3$, upper layer).

\section{Isolation and purification of the chemical constituents}

The powder of the aerial parts $(2 \mathrm{~kg})$ was extracted 3 times at room temperature with $70 \%$ methanol. The aqueous-methanol extract was evaporated under reduced pressure and temperature to obtain a residue of $350 \mathrm{~g}$. The residue was defatted by $\mathrm{n}$-hexane giving ( $80 \mathrm{~g}$ ) $\mathrm{n}$-hexane extract and $(270 \mathrm{~g})$ methanol extract.

\section{Fractionation of lipid constituents}

A fraction (1 g) of the n-hexane extract was analyzed for volatile constituents by GC/MS and after then, the rest of the n-hexane extract was passed over fuller's earth (to remove the colored pigments). The solvent was evaporated under reduced pressure at $35^{\circ} \mathrm{C}$; the obtained residue $(20 \mathrm{~g})$ was saponified to give unsaponifiable matters and the fatty acid methyl esters [19], which were analyzed by GC/MS and/or gas-liquid chromatography (GLC) as follows.

\section{GC/MS analysis of volatile constituents of $n$-hexane extract}

The analysis of n-hexane fraction of S. montana was performed using a Thermo Scientific capillary gas chromatography (model Trace GC ULTRA) directly coupled to ISQ Single Quadruple MS and equipped with TG-5MS, non-polar 5\% phenyl methylpolysiloxane capillary column $(30 \mathrm{~m} \times 0.25 \mathrm{~mm}$ ID $\times 0.25 \mu \mathrm{m})$. The operating condition of GC oven temperature was maintained as initial temperature $40^{\circ} \mathrm{C}$ for $3 \mathrm{~min}$, programmed rate $5^{\circ} \mathrm{C} / \mathrm{min}$ up to final temperature $280^{\circ} \mathrm{C}$ with isotherm for $5 \mathrm{~min}$. For GC/MS detection, an electron ionization (EI) system with ionization energy of $70 \mathrm{eV}$ was used. Helium was used as a carrier gas at a constant flow rate of $1.0 \mathrm{ml} / \mathrm{min} .1 \mu \mathrm{l}$ of the extract was injected automatically in the splitless mode. The quantification of the components was based on the total number of fragments (total ion count) of the metabolites as detected by the mass spectrometer. Identification of the constituents was carried out by comparison of their retention times and fragmentation patterns of mass with those of published data and/or with those of the Wiley and NIST mass spectra libraries.

GLC analysis of unsaponifiable matters and GC/MS of fatty acid methyl esters

The GLC analysis was carried out for unsaponifiable matters using the following conditions; Instrument: Varian model 3700 GC. Column for unsap.: 10\% OV-101 on chromosorb W/HP, 80/100, (2 m stainless steel, $0.25 \mathrm{~mm}$ i.d.), column for unsap.: Column: $70^{\circ} \mathrm{C}$ up to $270^{\circ} \mathrm{C}, 4^{\circ} \mathrm{C} /$ min., injector: $280^{\circ} \mathrm{C}$., detector (FID): $290^{\circ} \mathrm{C}$., temperature for unsap.: Column: $70^{\circ} \mathrm{C}$ up to $270^{\circ} \mathrm{C}, 4^{\circ} \mathrm{C} /$ min., injector: $280^{\circ} \mathrm{C}$, detector (FID): $290^{\circ} \mathrm{C}$.
While the conditions for FAMEs are instrument: A TRACE GC ultragas chromatographs (THERMO Scientific Corp., USA) coupled with a thermo mass spectrometer detector (ISQ Single Quadrupole Mass Spectrometer). The GC-MS system was equipped with a TG-5MS column ( $30 \mathrm{~m} \times 0.25 \mathrm{~mm}$ i.d., $0.25 \mu \mathrm{m}$ film thickness). Analyses were carried out using helium as carrier gas at a flow rate of $1.0 \mathrm{~mL} / \mathrm{min}$ and a split ratio of $1: 10$ using the following temperature program: $80^{\circ} \mathrm{C}$ for $1 \mathrm{~min}$; rising at $4.0^{\circ} \mathrm{C} / \mathrm{min}$ to $300^{\circ} \mathrm{C}$ and held for $1 \mathrm{~min}$. The injector and detector were held at $240^{\circ} \mathrm{C}$. Diluted samples $(1: 10$ hexane, v/v) of $0.2 \mu \mathrm{L}$ of the mixtures were always injected. Mass spectra were obtained by EI at 70 $\mathrm{eV}$, using a spectral range of $\mathrm{m} / \mathrm{z} 40-450$.

\section{Isolation of the phenolic constituents}

The methanol extract was subjected on polyamide S6 CC, eluting with $\mathrm{H}_{2} \mathrm{O}$ followed by $\mathrm{MeOH} / \mathrm{H}_{2} \mathrm{O}$ mixtures of decreasing polarity which yielded four fractions 40,60, 80, and 100\% methanol. Each fraction was subjected to further purification using columns chromatography (small polyamide and Sephadex LH-20) and preparative paper chromatography (Whatman 3MM, with different solvent systems). The fraction $40 \% \mathrm{MeOH} / \mathrm{H}_{2} \mathrm{O}$ yielded compound 1 (14 mg), followed by the fraction $60 \% \mathrm{MeOH} / \mathrm{H}_{2} \mathrm{O}$ which yielded compound $2(16 \mathrm{mg})$, compound 3 (17 mg), compound 4 (13 mg), and compound 5 (15 mg). On the other hand, compound $6(13 \mathrm{mg})$ and compound 7 (18 $\mathrm{mg})$ were isolated from the fraction $80 \% \mathrm{MeOH} / \mathrm{H}_{2} \mathrm{O}$, while $100 \% \mathrm{MeOH}$ fraction yielded compound 8 (19 mg).

\section{Acid hydrolysis}

It was carried out for $2 \mathrm{~h}$ at $100^{\circ} \mathrm{C}$ using $2 \mathrm{~N} \mathrm{HCl}$; the hydrolyzed part was then extracted with ethyl acetate; the extract being subjected to PC investigation to detect the aglycones. Sugars were identified by PC using (benzene:n-butanol:pyridine:water, 1:5:3:3) with authentic sugars. The dried chromatograms were visualized by aniline phthalate reagent, the sugar spots were observed in daylight. The tested sugars were compared with reference sugars. Identification of the aglycones was based on direct PC using authentic samples and/or spectral analyses.

\section{Antimicrobial activity study}

The antimicrobial activity of the tested extracts and/or isolated compounds against some microorganisms was determined using a modified Kirby-Bauer disc diffusion method [20-22].

Plates inoculated with filamentous fungi as Aspergillus flavus at $25^{\circ} \mathrm{C}$ for $48 \mathrm{~h}$; Gram (+) bacteria as Staphylococcus aureus and Bacillus subtilis; Gram (-) bacteria as Escherichia coli and Pseudomonas aeruginosa, they were incubated at $35-37^{\circ} \mathrm{C}$ for $24-48 \mathrm{~h}$ and yeast as Candida albicans incubated at $30^{\circ} \mathrm{C}$ for $24-48 \mathrm{~h}$, and then, the diameters of the inhibition zones were measured in millimeters [20]. The diameter of the inhibition zone surrounding the sample is taken as a measure of the inhibitory power of the sample against the particular test organism. Standard discs of ampicillin (antibacterial agent), amphotericin B (antifungal agent) served as positive controls for antimicrobial activity.

\section{Calculations}

$\%$ inhibition $=$ sample inhibition zone $(\mathrm{cm}) /$ plate diameter $\times 100$. The filter discs impregnated with $10 \mu$ of solvent (distilled water, chloroform, DMSO) were used as a negative control. The test was carried out in triplicates [23].

Antioxidant activity using 2,2-diphenyl-1-picrylhydrazyl (DPPH) radical-scavenging method

DPPH scavenging activity of the tested extracts and/or isolated compounds was measured at $517 \mathrm{~nm}$ by spectrophotometer method [24]. A $1 \mathrm{~mL}$ solution of $0.004 \%$ DPPH solution was added to the tested extracts and/or isolated compounds. The solution in the test tubes was shaken well and incubated in the dark for $30 \mathrm{~min}$ at room temperature. The disappearance of violet color of methanolic DPPH solution indicates scavenging capacity of the extract considered as the positive reaction. 


\section{RESULTS AND DISCUSSION}

The GC/MS analysis was used to identify the volatile constituents from n-hexane fraction of $S$. montana, 39 compounds belonging to many classes such as monoterpenes (30.7\%) with carvacrol as the major one $(24.23 \%)$, sesquiterpenes $(4.06 \%)$ in which farnesyl acetone is the main, diterpenes (17.67\%), one saturated fatty acid (palmitic acid, $17.1 \%)$, esters of fatty acids (10.14\%) with methyl hexadecatrienoate as a main ester, phthalates constituted about $2.9 \%$, and a sterol fraction of about $2.7 \%$ were identified (Table 1 ). These data were in accordance with that reported [15], where they identified the major constituents of hydrodistilled volatiles from the aerial parts of S. montana growing in Croatia and they found carvacrol $(17.7 \%)$ as the major compound. Furthermore, Hanene et al., 2013, identified carvacrol (53.17\%) as a main in the winter S. montana [25].

The fatty acids (Table 2) were found to contain a mixture of nine fatty acids from which six acids were identified and constituted about $91.28 \%$. The saturated fatty acids four acids with palmitic acid as major (40.19\%) and the unsaturated acids constitute about $47.64 \%$, in which linolenic acid is the main acid. These data are very near from that reported by Ahmet et al., in 2003 [19], where they studied the fatty

Table 1: GC/MS analysis of n-hexane fraction of S. montana

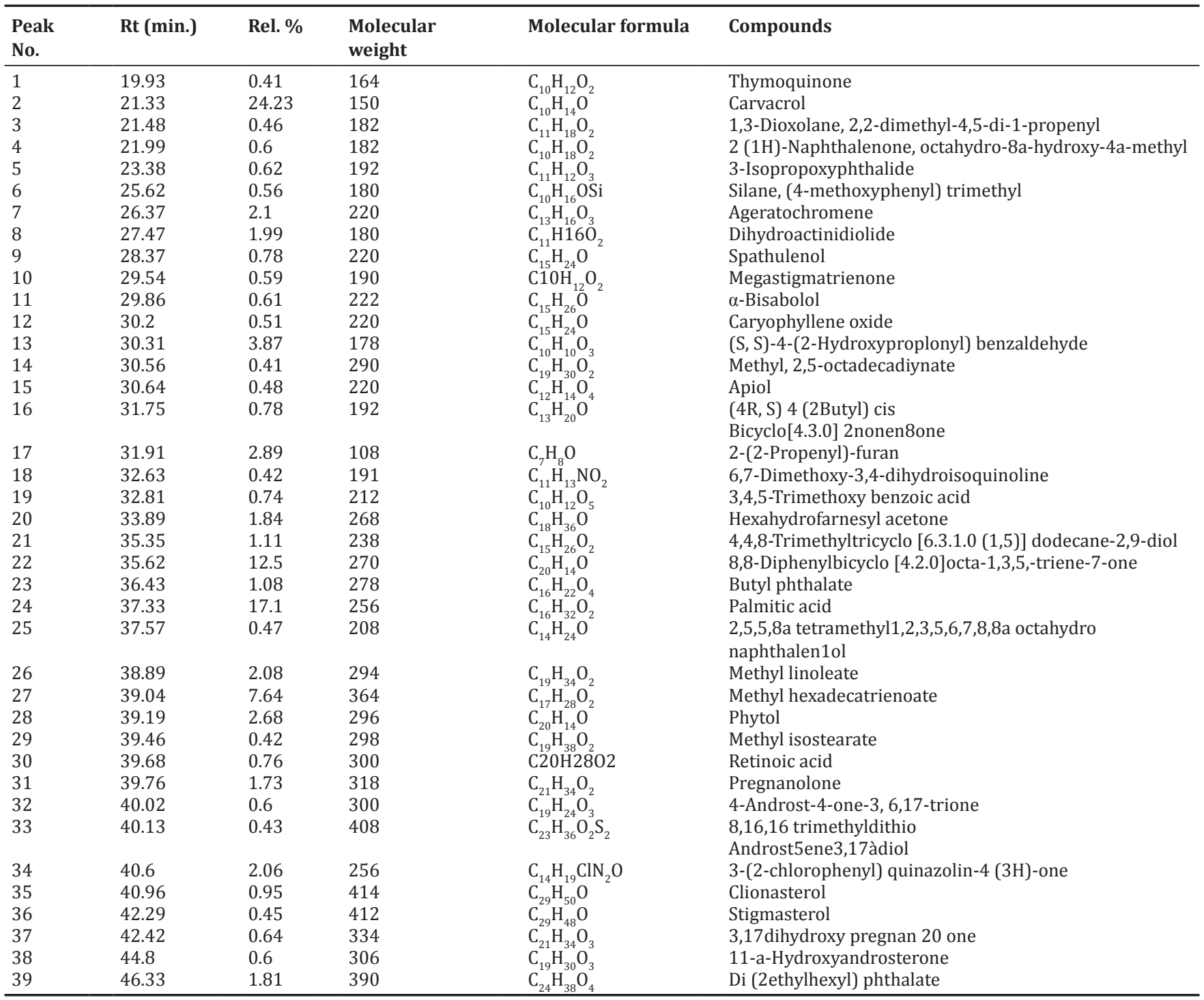

GC-MS: Gas chromatography-mass spectrometry, S. montana: Satureja montana

Table 2: GC/MS data of FAME of S. montana

\begin{tabular}{lllll}
\hline Peak No. & Rt (min.) & Rel. \% & Mol. Formula & Mol. Wt. \\
\hline 1 & 15.86 & 40.19 & $\mathrm{C}_{17} \mathrm{H}_{34} \mathrm{O}_{2}$ & 270 \\
2 & 19.74 & 5.68 & $\mathrm{C}_{19} \mathrm{H}_{34} \mathrm{O}_{2}$ & 294 \\
3 & 19.98 & 40.59 & $\mathrm{C}_{19} \mathrm{H}_{32} \mathrm{O}_{2}$ & Methyl Palmitate \\
4 & 20.44 & 3.01 & $\mathrm{C}_{19} \mathrm{H}_{38} \mathrm{O}_{2}$ & Methyl Linolelaidate \\
5 & 24.77 & 1.37 & $\mathrm{C}_{21} \mathrm{H}_{42} \mathrm{O}_{2}$ & Methyl Linolenate \\
6 & 28.78 & $\mathrm{C}_{23} \mathrm{H}_{46} \mathrm{O}_{2}$ & 326 \\
7 & 8.72 & Unknowns & 354 & Methyl Stearate \\
\end{tabular}


acids in $S$. thymbra and $S$. cuneifolia and found both palmitic acid and linolenic acid are major in both species.

The GLC analysis (Table 3) proved that the unsaponifiable fraction consists of a mixture of hydrocarbons (89.97\%) in which n-tricosane is the most abundant (27.7\%) and a sterol fraction of $10.03 \%$ with stigmasterol as a main.

Identification of the phenolic compounds: Investigation of the phenolic constituents resulted in isolation of seven flavonoidal compounds, in addition to gallic acid, their structures were carried out through color reactions, $\mathrm{R}_{\mathrm{f}}$ values, chemical investigations (acid hydrolysis), and spectral measurements (UV spectral analysis in methanol and on addition of shift reagents confirmed the aglycone and glycosidic linkage, $1 \mathrm{D}$ and $2 \mathrm{D}^{1} \mathrm{H}$ NMR, ${ }^{13} \mathrm{C}-\mathrm{NMR}$ ). Further, authentication was carried out by comparison of their spectroscopic data with previously published values. These compounds were identified as luteolin-7-rhamnoside4'-O- $\beta$-glucopyranoside (1), quercetin-3-O- $\alpha$-L-rhamnopyranoside (2), quercetin-7-O-glucopyranoside (3), luteolin-7-O-glucopyranoside (4), 5-hydroxy-6,7,8,4'-tetramethoxy flavone (5), gallic acid (6), 2,3-hexahydroxydiphenoyl 1-galloyl glucopyranoside (7), and quercetin (8). Complete acid hydrolysis indicated that compounds 1-4 and 7 were in $O$-glycosidic linkage $[26,27]$ as follows.

Compound (1): Luteolin-7-rhamnoside-4'-0- $\beta$-glucopyranoside: It was obtained as yellow amorphous powder $(14 \mathrm{mg})$, m.p. $320-324^{\circ} \mathrm{C}$. UV spectral data $\lambda_{\text {max }}(\mathrm{nm}) \mathrm{MeOH}: 270,340 ; \mathrm{NaOMe}: 270,377 ; \mathrm{AlCl}_{3:} 276$, 300sh, 348, 388; $\mathrm{AlCl}_{3} / \mathrm{HCl}$ : 277, 300sh, 348, 388; NaOAc: 269, 337, 348; $\mathrm{NaOAc} / \mathrm{H}_{3} \mathrm{BO}_{3:} 269,237,339 .{ }^{1} \mathrm{H}-\mathrm{NMR}\left(600 \mathrm{MHz}, \mathrm{DMSO}-\mathrm{d}_{6}\right): \delta(\mathrm{ppm})$ at 7.49, (1H, dd, J=8 Hz, H-6'), 7.51 (1H, d, J=2 Hz, H2'), 7.25 (1H, d, J=8 Hz, H-5'), 6.56 (1H, s, H-3), 6.87 (1H, d, J=2.5 Hz, H-8), $6.55(1 \mathrm{H}, \mathrm{d}, \mathrm{J}=2.5 \mathrm{~Hz}$, $\mathrm{H}-6)$. Sugar moiety, at $5.5(1 \mathrm{H}, \mathrm{d}, \mathrm{J}=7.5 \mathrm{~Hz}, \mathrm{H}-1$ " of glucose $), 4.55(1 \mathrm{H}, \mathrm{d}$, $\mathrm{J}=2 \mathrm{~Hz}, \mathrm{H}-1$ "' of rhamnose), $3.01 \rightarrow 3.5$ (complex signals, $\mathrm{m}$, due to rest sugar protons), $0.98\left(3 \mathrm{H}, \mathrm{d}, \mathrm{J}=6.1 \mathrm{~Hz}, \mathrm{CH}_{3}\right.$ of rhamnose moiety).

${ }^{13} \mathrm{C}$-NMR spectral measurement (150 MHz, DMSO-d ): Aglycone moiety $\delta(\mathrm{ppm})$ at 161.92 (C-2), 104.20 (C-3), 182.3 (C-4), 163.9 (C-5), 98.3 (C-6), 165.5 (C-7), 94.49 (C-8), 157.78 (C-9), 104.3 (C-10), 121.5 (C-1'), 118.64 (C-2'), 147.90 (C-3'), 151.98 (C-4'), 116.23 (C-5'), 124.70 (C-6'). Sugar moiety: 4'-O-glucoside, at 100.9 (C-1"), 72.49 (C-2"), 77.3 (C-3"), 71.1 (C-4"), 77.4 (C-5"), 60.07 (C-6"). 7-O-rhamnoside, at 100.1 (C-1"'), 70.3 (C-2'”), 70.7 (C-3'”'), 72.01 (C-4"'), 69.07 (C-5'”), and 18.4 (C-6"').

Complete acid hydrolysis yielded luteolin, glucose, and rhamnose, which was compared with authentic samples. Hence, the compound 1 was identified as luteolin-7-rhamnoside-4'-0- $\beta$-glucopyranoside.

Table 3: GLC data of unsaponifiable of $S$. montana

\begin{tabular}{lllll}
\hline $\begin{array}{l}\text { Peak } \\
\text { No. }\end{array}$ & Rt (min.) & Rel. \% & $\begin{array}{l}\text { Molecular } \\
\text { formula }\end{array}$ & Compounds \\
\hline 1 & 6.48 & 2.87 & $\mathrm{C}_{9} \mathrm{H}_{20}$ & n-nonane \\
2 & 9.72 & 1.58 & $\mathrm{C}_{11} \mathrm{H}_{24}$ & n-undecane \\
3 & 11.96 & 0.84 & $\mathrm{C}_{15} \mathrm{H}_{32}$ & n-pentadecane \\
4 & 13.29 & 4.98 & $\mathrm{C}_{17} \mathrm{H}_{36}$ & n-heptadecane \\
5 & 15.42 & 11.27 & $\mathrm{C}_{18} \mathrm{H}_{38}$ & n- octadecane \\
6 & 16.46 & 0.9 & $\mathrm{C}_{19} \mathrm{H}_{40}$ & n-nonadecane \\
7 & 17.36 & 12.32 & $\mathrm{C}_{20} \mathrm{H}_{42}$ & n-eicosane \\
8 & 18.45 & 9.46 & $\mathrm{C}_{21} \mathrm{H}_{44}$ & n-heneicosane \\
9 & 19.82 & 4.53 & $\mathrm{C}_{22} \mathrm{H}_{46}$ & n-docosane \\
10 & 20.03 & 27.7 & $\mathrm{C}_{23} \mathrm{H}_{48}$ & n-tricosane \\
11 & 21.39 & 4.99 & $\mathrm{C}_{24} \mathrm{H}_{50}$ & n-tetracosane \\
12 & 22.71 & 1.26 & $\mathrm{C}_{25} \mathrm{H}_{52}$ & n-pentacosane \\
13 & 23.47 & 6.12 & $\mathrm{C}_{26} \mathrm{H}_{54}$ & n-hexacosane \\
14 & 24.41 & 1.15 & $\mathrm{C}_{27} \mathrm{H}_{56}$ & n-heptacosane \\
15 & 26.01 & 0.98 & $\mathrm{C}_{27} \mathrm{H}_{46} \mathrm{O}$ & Cholesterol \\
16 & 27.51 & 1.18 & $\mathrm{C}_{28} \mathrm{H}_{48} \mathrm{O}$ & Campasterol \\
17 & 28.04 & 5.64 & $\mathrm{C}_{29} \mathrm{H}_{48} \mathrm{O}$ & Stigmasterol \\
18 & 29.95 & 2.23 & $\mathrm{C}_{29} \mathrm{H}_{50} \mathrm{O}$ & $\beta$-Sitosterol \\
\hline S. montana: Satureja montana $\mathrm{GLC}$ - Gas-liquid chromatography
\end{tabular}

S. montana: Satureja montana, GLC: Gas-liquid chromatography
Compound (2): Quercetin-3-O- $\alpha$-L-rhamnopyranoside: Yellow amorphous powder $(16 \mathrm{mg})$, m.p. $316-318^{\circ} \mathrm{C}$. UV spectral data $\lambda_{\max }$ (nm) MeOH: 258, 269sh, 360; NaOMe: 272, 328sh, 405; $\mathrm{AlCl}_{3:} 275$, 305sh, 332sh, 435; $\mathrm{AlCl}_{3} / \mathrm{HCl}: 275$, 305sh, 361, 403; NaOAc: 269, 323sh, 380; $\mathrm{NaOAc} / \mathrm{H}_{3} \mathrm{BO}_{3}$. 262, 300sh, 387; ${ }^{1} \mathrm{H}-\mathrm{NMR}$ (600 MHz, DMSO-d ${ }_{6}$ ): Aglycone moiety: $\delta$ ppm: $7.6\left(1 \mathrm{H}, \mathrm{d}, \mathrm{J}=2.5 \mathrm{~Hz}, \mathrm{H}-2^{2}\right), 7.5(1 \mathrm{H}, \mathrm{dd}, \mathrm{J}=2.5 \mathrm{~Hz}$ and $\left.\mathrm{J}=8 \mathrm{~Hz}, \mathrm{H}-6^{\prime}\right), 6.77\left(1 \mathrm{H}, \mathrm{d}, \mathrm{J}=8 \mathrm{~Hz}, \mathrm{H}-5^{\prime}\right), 6.24(1 \mathrm{H}, \mathrm{d}, \mathrm{J}=2.0 \mathrm{~Hz}, \mathrm{H}-8)$, $6.04(1 \mathrm{H}, \mathrm{d}, \mathrm{J}=2.0 \mathrm{~Hz}, \mathrm{H}-6) \mathrm{ppm}$. Sugar moiety: $5.5(1 \mathrm{H}, \mathrm{d}, \mathrm{J}=2.0 \mathrm{~Hz}$, $\mathrm{H}-1$ " of rhamnose), $3.1 \rightarrow 3.5$ (m, sugar protons), $0.98(3 \mathrm{H}, \mathrm{d}, \mathrm{J}=6.1 \mathrm{~Hz}$, $\mathrm{CH}_{3}$ of rhamnosyl) ppm. ${ }^{13} \mathrm{C}-\mathrm{NMR}\left(150 \mathrm{MHz}\right.$, DMSO-d $\mathrm{d}_{6}$ ): 157.04 (C-2), 134.5 (C-3), 177.9 (C-4), 161.7 (C-5), 99.5 (C-6), 161.2 (C-7), 94.3 (C8), 157.4 (C-9), 103.9 (C-10), 121.1 (C-1'), 115.9 (C-2'), 145.76 (C-3'), 149.1 (C-4'), 115.99 (C-5'), 121.52 (C-6'). Sugar moiety: 101.1 (C-1"), 70.6 (C-2"), 70.81 (C-3"), 71.66 (C-4"), 70.5 (C-5"), and 17.9 (C-6"). The acid hydrolysis of compound 2 yielded both quercetin and rhamnose, which was identified by Co-PC, using authentics in different solvents. Thus, the structure of compound 2 was proved as quercetin-3-0- $\alpha-\mathrm{L}-$ rhamnopyranoside.

Compound (3): Quercetin-7-0-glucopyranoside: The compound was obtained as yellow amorphous powder $(17 \mathrm{mg})$, m.p. $318-320^{\circ} \mathrm{C}$. UV spectral data $\lambda_{\text {max }}(\mathrm{nm}) \mathrm{MeOH}: 257,294$ sh, 357; NaOMe: 266, 314, 406; $\mathrm{AlCl}_{3}$ 273, 301sh, 335sh, 433; $\mathrm{AlCl}_{3} / \mathrm{HCl}: 269,300 \mathrm{sh}, 358,402 ; \mathrm{NaOAc}$ : 259, 396sh, 367, 415; NaOAc/ $\mathrm{H}_{3} \mathrm{BO}_{3} 261,295 \mathrm{sh}, 378 .{ }^{1} \mathrm{H}-\mathrm{NMR}(600$ MHz, DMSO- $\left.\mathrm{d}_{6}\right): \delta(\mathrm{ppm})$ at $12.60(1 \mathrm{H}, \mathrm{s}, \mathrm{OH}-5), 7.61(1 \mathrm{H}, \mathrm{d}, \mathrm{J}=2.2 \mathrm{~Hz}$, $\left.\mathrm{H}-2^{\prime}\right), 7.58\left(1 \mathrm{H}, \mathrm{dd}, \mathrm{J}=2.2,8.0 \mathrm{~Hz}, \mathrm{H}-6^{\prime}\right), 6.86\left(1 \mathrm{H}, \mathrm{d}, \mathrm{J}=8 \mathrm{~Hz}, \mathrm{H}-5^{\prime}\right)$, $6.7(1 \mathrm{H}, \mathrm{d}, \mathrm{J}=2.0 \mathrm{~Hz}, \mathrm{H}-8), 6.48(1 \mathrm{H}, \mathrm{d}, \mathrm{J}=2.0 \mathrm{~Hz}, \mathrm{H}-6)$. The sugar protons at $5.5(1 \mathrm{H}, \mathrm{J}=7.5 \mathrm{~Hz}, \mathrm{H}-1$ "), $3.1 \rightarrow 3.6$ (complex m).

${ }^{13}$ C-NMR: 159.1 (C-2), 133.6 (C-3), 177.4 (C-4), 162.2 (C-5), 98.6 (C-6), 165.6 (C-7), 94.4 (C-8), 159.1 (C-9), 104.6 (C-10), 122.1 (C-1'), 116.1 (C-2'), 145.2 (C-3'), 150.2 (C-4'), 116.9 (C-5'), 122.7 (C-6'), 100.6 (glc, C-1), 74.2 (glc, C-2), 77.9 (glc, C-3), 70.2 (glc, C-4), 76.4 (glc, C-5), 61.7 (glc, C-6). Complete acid hydrolysis of compound 3 gave quercetin and glucose by comparing with Co-PC with authentic samples. Compound 3 was identified as quercetin-7-0-glucopyranoside.

Compound (4): Luteolin-7-0-glucopyranoside: Off white amorphous powder (13 mg), m.p.324-326 ${ }^{\circ} \mathrm{C}$. UV spectral data $\lambda_{\text {myx }}(\mathrm{nm}) \mathrm{MeOH}: 258$, 347; NaOMe: 262.5, 298sh, 398; $\mathrm{AlCl}_{3 .} 275,302,345,385,421 ; \mathrm{AlCl}_{3} /$ HCl: 269, 280, 295sh, 340, 282, 387; NaOAc: 267, 379, 423; NaOAc/ $\mathrm{H}_{3} \mathrm{BO}_{3 .} 261.5,370 .{ }^{1} \mathrm{H}-\mathrm{NMR}$ (DMSO- $\left.\mathrm{d}_{6}\right): 7.7(1 \mathrm{H}, \mathrm{dd}, \mathrm{J}=2.5 \mathrm{~Hz}$, and $\mathrm{J}=8 \mathrm{~Hz}$, H-6'), 7.55 (1H, d, J=2.5 Hz, H-2'), 6.85 (1H, d, J=8 Hz, H-5'), 6.42 (1H, s, $\mathrm{H}-3), 6.72(1 \mathrm{H}, \mathrm{d}, \mathrm{J}=2.0 \mathrm{~Hz}, \mathrm{H}-8), 6.61(1 \mathrm{H}, \mathrm{d}, \mathrm{J}=2.0 \mathrm{~Hz}, \mathrm{H}-6), 5.2(1 \mathrm{H}, \mathrm{d}$, $\mathrm{J}=7.5 \mathrm{~Hz}, \mathrm{H}-1$ "), $3.2 \rightarrow 3.8$ (m, of sugar protons). ${ }^{13} \mathrm{C}-\mathrm{NMR}: 161.5$ (C-2), 103.5 (C-3), 182.3 (C-4), 163.39 (C-5), 99.9 (C-6), 164.5 (C-7), 95.16 (C-

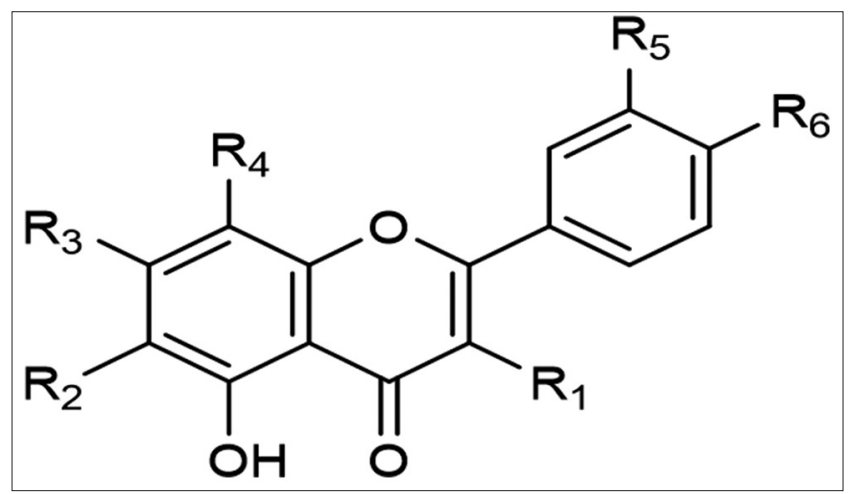

Fig.1: Phenolic compounds isolated from $S$. montana $\mathrm{L}$

Compound 1 R1, R2, R4 = H, R3= 0-rhamnose, R5=0H, R6 =0-glucose

Compound 2 R1=0-rhamnose, R2,R4= H, R3, R5, R6= OH

Compound 3 R1, R5, R6 = OH, R2, R4 = H, R3= 0-glucose

Compound $4 \quad \mathrm{R} 1, \mathrm{R} 2, \mathrm{R} 4=\mathrm{H}, \mathrm{R} 3=0$-glucose, $\mathrm{R5}, \mathrm{R} 6=\mathrm{OH}$

Compound 5 R1, R5 = H, R2, R3, R4, R6 = OCH3

Compound 8 R1, R3, R5, R6 = OH, R2, R4 = H 
Table 4: Antimicrobial activity of $S$. montana (Dry DMSO as solvent)

\begin{tabular}{|c|c|c|c|c|c|c|}
\hline Sample name & Inhibition & eter in $\mathbf{~ m m}$ & & & & \\
\hline \multirow[t]{2}{*}{$\begin{array}{l}\text { n-Hexane } \\
\text { extract }\end{array}$} & $\begin{array}{l}\text { B. subtilis } \\
(\mathrm{G}+)\end{array}$ & $\begin{array}{l}\text { S. aureus } \\
(\mathrm{G}+)\end{array}$ & $\begin{array}{l}\text { E. coli } \\
\text { (G-) }\end{array}$ & $\begin{array}{l}\text { P. aeruginosa } \\
\text { (G-) }\end{array}$ & $\begin{array}{l}\text { A. flavus } \\
\text { (Fungus) }\end{array}$ & $\begin{array}{l}\text { C. albicans } \\
\text { (Fungus) }\end{array}$ \\
\hline & 12 & 10 & 11 & 11 & 5 & 6 \\
\hline Methanol extract & 21 & 18 & 20 & 21 & 10 & 12 \\
\hline Compound (1) & 15 & 16 & 16 & 17 & 7 & 8 \\
\hline Compound (2) & 22 & 19 & 21 & 22 & 10 & 11 \\
\hline Compound (4) & 20 & 17 & 19 & 20 & 8 & 9 \\
\hline Compound (5) & 14 & 15 & 14 & 15 & 5 & 7 \\
\hline Compound (6) & 13 & 12 & 13 & 14 & 6 & 7 \\
\hline Compound (7) & 14 & 13 & 13 & 14 & 7 & 9 \\
\hline Compound (8) & 21 & 17 & 19 & 20 & 9 & 10 \\
\hline Ampicillin & 26 & 21 & 25 & 26 & - & - \\
\hline Amphotericin B & - & - & - & - & 16 & 19 \\
\hline
\end{tabular}

P. aeruginosa: Pseudomonas aeruginosa, A. flavus: Aspergillus flavus, B. subtilis: Bacillus subtilis, S. aureus: Staphylococcus aureus, E. coli: Escherichia coli, C. albicans: Candida albicans

8), 157.4 (C-9), 105.78 (C-10), 121.66 (C-1'), 119.6(C-2'), 146.3 (C-3'), 150.4 (C-4'), 116.42 (C-5'), 122.1 (C-6'). Sugar moiety $\delta$ ppm: 100.3 (C1"), 72.3 (C-2"), 77.6 (C-3"), 69.99 (C-4"), 76.83 (C-5"), 61.06 (C-6"). Acid hydrolysis of compound 4 led to identification of both luteolin and glucose by Co-PC using authentic markers in different solvents. The compound 4 could be identified as luteolin-7-0-glucopyranoside.

Compound (5): 5-Hydroxy-6,7,8,4'-tetramethoxy flavone: It was obtained as white yellowish amorphous powder (15 mg), m.p. 224$226^{\circ} \mathrm{C}$. UV spectral data $\lambda_{\max }(\mathrm{nm}) \mathrm{MeOH}: 254 \mathrm{sh}, 277,342$, NaOMe: 268 , 406; $\mathrm{AlCl}_{3 .} 272 \mathrm{sh}, 287,309,362,410 \mathrm{sh} ; \mathrm{AlCl}_{3} / \mathrm{HCl}$ : 262sh, 287, 310, 360, 408sh; NaOAc: 259sh, 276, 342, 406; $\mathrm{NaOAc} / \mathrm{H}_{3} \mathrm{BO}_{3}$ 254sh, 277, 339; ${ }^{1} \mathrm{H}-\mathrm{NMR}(600 \mathrm{MHz}$, DMSO-d $): \delta(\mathrm{ppm})$ at $7.9(2 \mathrm{H}, \mathrm{dd}, \mathrm{J}=2.5 \mathrm{~Hz}, 8 \mathrm{~Hz}$, H-2' and H-6'), 7.01 (2H, dd, J=2.5 Hz, $8 \mathrm{~Hz}$ for $\mathrm{H}-3^{\prime}$ and $\left.\mathrm{H}-5^{\prime}\right), 6.9(1 \mathrm{H}$, $\mathrm{s}, \mathrm{H}-3), 4.1\left(\mathrm{~s}, \mathrm{OCH}_{3}-4^{\prime}\right), 4.02\left(\mathrm{~s}, \mathrm{OCH}_{3}-6\right), 3.9\left(\mathrm{~s}, \mathrm{OCH}_{3}-7\right), 3.85\left(\mathrm{~s}, \mathrm{OCH}_{3}-\right.$ 8). ${ }^{13}$ C-NMR: 162 (C-2), 103.1 (C-3), 183.02 (C-4), 148.54 (C-5), 136.30 (C-6), 152.92 (C-7), 133.07 (C-8), 145.68 (C-9), 106.66 (C-10), 121.50 (C-1'), 110.48 (C-2'), 116.4 (C-3'), 154.6 (C-4'), 116.40 (C-5'), 128.98 (C-6'), $62.31\left(\mathrm{OCH}_{3}-6\right), 62.37\left(\mathrm{OCH}_{3}-7\right), 61.95\left(\mathrm{OCH}_{3}-8\right), 55.24\left(\mathrm{OCH}_{3}-\right.$ $\left.4^{\prime}\right)$. The above data showed that compound 5 is 5 -Hydroxy- $6,7,8,44^{\prime}-$ tetramethoxy flavone.

Compound (6): Gallic acid: White amorphous powder. m.p. $258-260^{\circ} \mathrm{C}$ UV spectral data $\lambda_{\max }(\mathrm{nm}) \mathrm{MeOH}: 272 \mathrm{~nm} .{ }^{1} \mathrm{H}-\mathrm{NMR}\left(600 \mathrm{MHz}\right.$, DMSO-d ) $^{2}$ $\delta(\mathrm{ppm})$ at $6.83\left(2 \mathrm{H}, \mathrm{s}, \mathrm{H}-2\right.$, and H-6). ${ }^{13} \mathrm{C}-\mathrm{NMR}$ at 120.6 (C- 1$), 108.8$ (C-2 and C-6), 145.5 (C-3 and C-5), 138.1 (C-4), 167.7 (C-7). Hence, compound 6 was identified as gallic acid [28].

Compound (7): 2,3-hexahydroxydiphenoyl 1-galloyl glucopyranoside; it was obtained as yellowish powder $(18 \mathrm{mg})$, m.p. $226-228^{\circ} \mathrm{C}$. UV spectral data $\lambda_{\text {max }}(\mathrm{nm}) \mathrm{MeOH}: 220,226,277 .{ }^{1} \mathrm{H}-\mathrm{NMR}$ : 7.1 (2H, s, H-2, 6 of galloyl proton), $6.8(1 \mathrm{H}, \mathrm{s}, \mathrm{H}-5$ of hexahydroxydiphenoyl portion (HHDP) ring C), $6.74(1 \mathrm{H}, \mathrm{s}$, HHDP $\mathrm{H}-5$ ring B), $6.4(1 \mathrm{H}, \mathrm{d}, \mathrm{J}=8 \mathrm{~Hz}$, glucose (Glc) H-1), $5.7(1 \mathrm{H}, \mathrm{d}, \mathrm{J}=8 \mathrm{~Hz}, \mathrm{Glc}, \mathrm{H}-2), 5.6(1 \mathrm{H}, \mathrm{d}, \mathrm{J}=8 \mathrm{~Hz}, \mathrm{Glc}$, $\mathrm{H}-3), 4.3(1 \mathrm{H}, \mathrm{d}, \mathrm{J}=8 \mathrm{~Hz}, \mathrm{Glc}, \mathrm{H}-4), 4.2(1 \mathrm{H}, \mathrm{t}, \mathrm{J}=9.4 \mathrm{~Hz}, \mathrm{Glc}, \mathrm{H}-5), 4.1(2 \mathrm{H}$, t, J=11, dd, J=2.8, 8 Hz, $\mathrm{CH}_{2}$-Glc). ${ }^{13} \mathrm{C}-\mathrm{NMR}: 93.95$ (Glc, C-1), 72.77 (Glc, C-2) 75.5 (Glc, C-3), 69.1 (Glc, C-4), 74.1 (Glc, C-5), 61.5 (Glc, C-6), 120.72 (gallic acid (Gal), C-1), 110.7 (Gal, C-2, 6), 145.71 (Gal, C-3, 5), 139.04 (Gal, C-4), 164.85 (Gal, $\mathrm{C}=0$ ), 115.76 (HHDP ring $\mathrm{B}, \mathrm{C}-1$ ), 116.27 (HHDP ring C, C-1), 144.55, 144.65, 144.66, 145.32 (HHDP ring $\mathrm{B}, \mathrm{C}-2$, 4; ring C, C-2, 4), 136.62 (HHDP ring $\mathrm{B}, \mathrm{C}-3$ ), 137.5 (HHDP ring C, C-3), 107.88 (HHDP ring B, C-5), 109.66 (HHDP ring C, C-5), 125.40 (HHDP ring $\mathrm{B}, \mathrm{C}-6$ ), 125.54 (HHDP ring $\mathrm{C}, \mathrm{C}-6$ ), 167.22 (HHDP ring $\mathrm{C}$, $\mathrm{C}=0$ ), 168.24 (HHDP ring $\mathrm{B}, \mathrm{C}=0$ ). Compound 7 was subjected to acid hydrolysis, yielding glucose, gallic acid, and hexahydroxydiphenic acid, the latter has high tendency to spontaneously undergo lactonization to afford ellagic acid (EA) $[27,28]$ and identified by Co-PC using authentic markers in different solvents. Hence, compound 7 could be identified as 2,3-hexahydroxydiphenoyl-1-galloyl glucopyranoside.

Compound (8): Quercetin: Yellow crystals (19 mg), m.p. 322-324 ${ }^{\circ} \mathrm{C}$. UV spectral data $\lambda_{\max }(\mathrm{nm}) \mathrm{MeOH}: 256,268 \mathrm{sh}, 371$; NaOMe: 249, 424; $\mathrm{AlCl}_{3:}$ 273, 305sh, 335sh; $\mathrm{AlCl}_{3} / \mathrm{HCl}$ : 266, 303sh, 350, 414; NaOAc: 264, 325, 390; NaOAc/ $\mathrm{H}_{3} \mathrm{BO}_{3} .262,302 \mathrm{sh}, 386 .{ }^{1} \mathrm{H}-\mathrm{NMR}$ ( $600 \mathrm{MHz}$, DMSO-d ${ }_{6}$ ): $7.8\left(1 \mathrm{H}, \mathrm{dd}, \mathrm{J}=2.5 \mathrm{~Hz}\right.$ and $\left.\mathrm{J}=8 \mathrm{~Hz}, \mathrm{H}-6^{\prime}\right), 7.6\left(1 \mathrm{H}, \mathrm{d}, \mathrm{J}=2.5 \mathrm{~Hz}, \mathrm{H}-2^{\prime}\right)$, $6.88\left(1 \mathrm{H}, \mathrm{d}, \mathrm{J}=8.5 \mathrm{~Hz}, \mathrm{H}-5^{\prime}\right), 6.4(1 \mathrm{H}, \mathrm{d}, \mathrm{J}=2.5 \mathrm{~Hz}, \mathrm{H}-8)$, and $6.2(1 \mathrm{H}, \mathrm{d}$, $\mathrm{J}=2.5 \mathrm{~Hz}, \mathrm{H}-6$ ) ppm. ${ }^{13} \mathrm{C}-\mathrm{NMR}: 147.25$ (C-2), 136.18 (C-3), 176.29 (C4), 161.17 (C-5), 98.63 (C-6), 164.35 (C-7), 93.81 (C-8), 156.59 (C-9), 103.46 (C-10), 122.40 (C-1'), 115.3 (C-2'), 154.51 (C-3'), 148.15 (C4'), 115.9 (C-5'), 120.43 (C-6'). From the above data, compound 8 was identified as quercetin $[26,27,29,30]$.

The antimicrobial activity of different extracts and isolated compounds

The antimicrobial activity of different extracts and isolated compounds data was summarized in Table 4, which proved that the methanolic extract showed significant antimicrobial activity against $S$. aureus (85.7\%), while, it showed a moderate antifungal activity against A. flavus (62.5\%) and C. albicans (63.2\%). On the other hand, the n-hexane extract showed a relatively low activity against Gram-positive B. subtilis (46.2\%), while, it showed a low antifungal activity against A. flavus (31.3\%).

Compound 2 showed the highest activity against Gram-positive B. subtilis and S. aureus ( $84.6 \%$ and $90.5 \%$ ), respectively. Compound 8 exhibited a significant inhibition against the tested Gram-positive B. subtilis and S. aureus (80.8\% and $81 \%)$, respectively, Gram-negative E. coli $(76 \%)$ and P. aeruginosa $(77 \%)$, while it showed a moderate antifungal activity against $A$. flavus and C. albicans (56.3\% and 52.6\%), respectively. Compound 4 exhibited a significant inhibition against $B$. subtilis (77\%), Gram-negative E. coli and P. aeruginosa (76\% and 77\%), respectively, and relatively moderate antifungal activity against $A$. flavus and C. albicans (50\% and $47.4 \%$ )

Furthermore, compound 3 exhibited a moderate activity against Gram-negative E. coli and P. aeruginosa (68\% and 69.2\%), respectively. Compounds 1 and 5 exhibited a significant inhibition against Grampositive S. aureus $(76.2 \%$ and $71.4 \%)$, respectively, and B. subtilis (57.7\% and 53.8\%), respectively, Gram-negative P. aeruginosa $(65.4 \%$ and $57.7 \%)$, respectively, and E. coli (64\% and $56 \%)$, respectively. On the other hand, both compounds showed a low antifungal activity against A. flavus (43.7\% and $31.2 \%$ ), respectively, and C. albicans $(42.1 \%$ and $36.8 \%)$, respectively. 


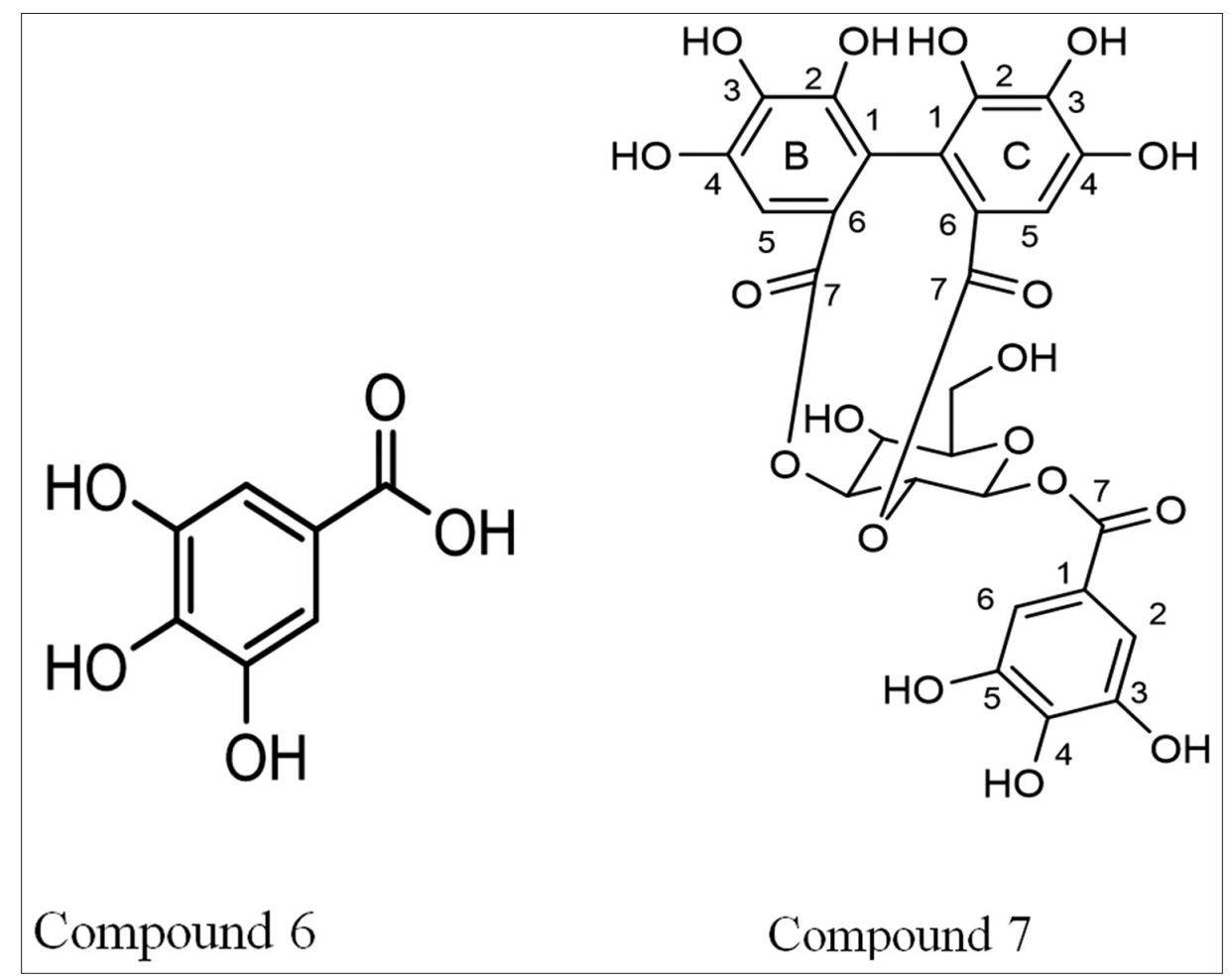

Fig. 2: Phenolic compounds isolated from Satureja montana L.

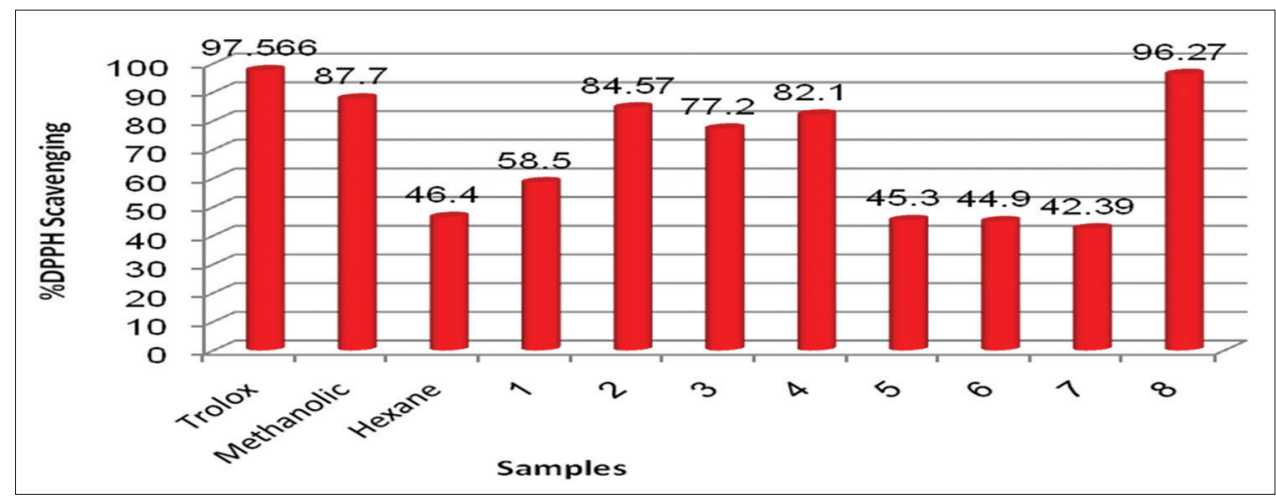

Fig. 3: Antioxidant activity of extracts and compounds from Satureja montana

Compounds 6 and 7 showed a moderate activity against Gram-positive S. aureus (57.1\% and 61.9\%), respectively, B. subtilis (50\% and $53.8 \%$ ), respectively, Gram-negative P. aeruginosa ( $53.9 \%$ both) and E. coli (52\% both).

The observed results in this study might be due to the presence of flavonoidal compounds in methanol extract and some volatile constituents beside the high percentage of unsaturated fatty acids in the hexane extract $[31,32]$.

\section{Antioxidant activity}

The antioxidant activity study (Fig. 3) proved that the compound 2 and the methanolic extract exhibited the highest activity (inhibition $\%=96.27$ and 87.7, respectively). On the other hand, n-hexane extract and compounds 5-7 showed low activity, while compounds $2-4$ gave moderate activity.

These data can be explained in light of the structure of different flavonoids play an important role in their activity as antioxidant where the structure of the flavonoids is the most significant determinant of radical scavenging $[33,34]$. The $\mathrm{OH}$ groups on the ring-B give hydrogen and an electron to $\mathrm{OH}$ and peroxyl radicals, which led to more flavonoidal radical stability and the activity increases linearly, according to the total number of $\mathrm{OH}$ groups [35]. When 3',4'-dihydroxy structure in ring-B, it increases lipid peroxidation inhibition effectively [36]. Hence, the peroxyl radical scavenging ability of luteolin substantially exceeds kaempferol [37]. Free radical scavenging of flavonoids is strongly dependent on the presence of a free $\mathrm{OH}$ group at $\mathrm{C}-3$ in the flavonoid nucleus [38]. Flavonoids with a C-3-OH and 3', ' $^{\prime}$ dihydroxy are reported to be 10 -fold more potent against peroxynitrite. The superiority of quercetin in inhibiting both metal and non-metal-induced oxidative damage is partially attributed to its free $3-0 \mathrm{H}$ substituent, which is thought to increase the stability of the flavonoid radical [39].

\section{CONCLUSION}

The findings of this study showed that the hydroalcoholic extract of S. montana showed significant antimicrobial and antioxidant activity. On the other hand, compound 2 showed the highest antibacterial activity against all the tested microorganisms, and compound 8 showed the highest antioxidant activity (96.27\%) in DPPH assay.

\section{CONFLICT OF INTEREST}

The authors declare that there are no conflicts of interests regarding the publication of this article. 


\section{AUTHOR CONTRIBUTIONS}

Ali M. El-Hagrassi, designed the experiments, extraction, separate pure compounds and lipid constituents and collect results, make the laboratory and store experiments and writing the manuscript. Walid E. Abdallah, share in isolation and separate pure compounds, volatile and lipid constituents, and writing the manuscript. Abeer F. Osman, share in extraction, isolation, separate pure compounds, volatile and lipid constituents, antioxidant and antimicrobial activity and writing the manuscript. Khaled A. Abdelshafeek, share in compounds identification, auditing, writing the research and reviewed the manuscript.

\section{REFERENCES}

1. Redžić S. Wild edible plants and their traditional use in the human nutrition in Bosnia-Herzegovina. Free Radic Res 2006;45:189-232.

2. Leporatti ML, Ivancheva S. Preliminary comparative analysis of medicinal plants used in the traditional medicine of Bulgaria and Italy. J Ethnopharmacol 2003;87:123-42.

3. Radonic A, Milos M. Chemical composition and in vitro evaluation of antioxidant effect of free volatile compounds from Satureja montana L. Free Radic Res 2003;37:673-9.

4. Skočibušić M, Bežić N. Chemical composition and antidiarrhoeal activities of winter savory (Satureja montana L.) essential oil. Pharm Biol 2003;41:622-6.

5. Skočibušić M, Bežić N. Chemical composition and antimicrobial variability of Satureja montana L. Essential oils produced during ontogenesis. J Essent Oil Res 2004;16:387-391

6. Oussalah M, Caillet S, Saucier L, Lacroix M. Inhibitory effects of selected plant essential oils on the growth of four pathogenic bacteria: E. Coli O157:H7, Salmonella typhimurium, St. aureus and Listeria monocytogenes. Food Control 2007;18:414-20.

7. Ćavar S, Maksimović M, Šolić M, Jerković-Mujkić E, Bešta R. Chemical composition and antioxidant and antimicrobial activity of two Satureja essential oils. Food Chem 2008;111:648-53.

8. Zavatti M, Zanoli P, Benelli A, Rivasi M, Baraldi C, Baraldi M, et al. Experimental study on Satureja montana as a treatment for premature ejaculation. J Ethnopharmacol 2011;133:629-33.

9. Haloc EI, Toska V, Baldisserotto A, Goci E, Vertuani S, Manfredini S. Evaluation of antifungal activity of Satureja montana essential oil before and after inclusion in beta-cyclodextrin. Int J Pharm Pharm Sci 2016;6(7):189-91

10. Gohari AR, Hadjiakhoondi A, Sadat-Ebrahimi SE, Saeidnia S, Shafiee A. Cytotoxic terpenoids from Satureja macrantha CA Mey. DARU 2005; $13: 177-81$

11. Moghaddam FM, Farimani MM, Salahvarzi S, Amin G. Chemical constituents of dichloromethane extract of cultivated Satureja khuzistanica. Evid Based Complement Alternat Med 2007;4:95-8.

12. Gohari AR, Saeidnia S, Gohari MR, Moradi-Afrapoli F, Malmir M, Hadjiakhoondi A, et al. Bioactive flavonoids from Satureja atropatana bonge. Nat Prod Res 2009;23:1609-14.

13. Saeidnia S, Nourbakhsh MS, Gohari AR, Davood A. Isolation and identification of the main compounds of Satureja sahendica Bornm. Aust J Basic Appl Sci 2011;5:1450-3.

14. Gohari AR, Ostad SN, Moradi-Afrapoli F, Malmir M, Tavajohi S, Akbari H. Evaluation of the cytotoxicity of Satureja spicigera and it's main compounds. Sci World J 2012;2012:203861.

15. Sanja Ć, Marija E Š, Milka M. Chemical composition and antioxidant activity of two Satureja species from Mt. Biokovo. Bot Serbia 2013;37:159-16

16. Malmir M, Gohari AR, Saeidnia S, Silva O. A new bioactive monoterpene-flavonoid from Satureja khuzistanica. Fitoterapia 2015; 105:107-12.

17. Ahamad RG, Abbas H, Seyed E, Sadat E, Soodabeh S, Abbas S. Cytoxic terpenoids from S. Macrantha. DARU 2005;13:4
18. Melpomeni S, Renee JG, Geoffrey C.K. Surface flavonoids in S. thymbra and S. spinosa (Lamiaceae). Biochem Syst Ecol 2005;33:541-4.

19. Gören AC, Bilsel G, Altun M, Satil F, Dirmenci T. Fatty acid composition of seeds of Satureja thymbra and S. cuneifolia. Z Naturforsch C 2003;58:502-4

20. Bauer AW, Kirby WM, Sherris C, Turck M. Antibiotic susceptibility testing by a standardized single disk method. Am J Clin Pathol 1966;45:493-6.

21. National Committee for Clinical Laboratory Standards. Reference Method for Broth Dilution Antifungal Susceptibility Testing of Conidium-Forming Filamentous Fungi: Proposed Standard M 38-A. Wayne, PA, USA: NCCLS; 2002.

22. National Committee for Clinical Laboratory Standards. Method for antifungal disk Diffusion Susceptibility Testing of Yeast: Proposed Guideline M44-P. Wayne, PA, USA: NCCLS; 2003.

23. Mohammed RS, El Souda SS, Taie HA, Moharam ME, Shaker KH. Antioxidant, antimicrobial activities of flavonoids glycoside from Leucaena leucocephala leaves. J Appl Pharm Sci 2015;5:138-47.

24. Braca A, De Tommasi N, Di Bari L, Pizza C, Politi M, Morelli I. Antioxidant principles from Bauhinia tarapotensis. J Nat Prod 2001;64:892-5.

25. Hanene M, Rihab BS, Donia M, Sami Z, Amina B, Emna A. Chemical composition and cytotoxic and antioxidant activities of Satureja montana L. Essential oil and Its antibacterial potential against Salmonella Spp. Strains J Chem 2013;2013:9.

26. Harbone JB. Phytochemical Methods. London: Chapman and Hall; 1984.

27. Mabry TJ, Markham KR, Thomas MB. The Systematic Identification of Flavonoids. New York: Springer-Verlag; 1970.

28. Nawwar MA, Hussein SA, Merfort I. NMR spectral analysis of polyphenenolics from Punica granatum. Phytochemistry 1994;36:793- 8 .

29. Luger P, Weber M, Kashino S, Amakura Y, Yoshida T, Okuda T, et al. Structure of the tannin geraniin based on conventional X-ray data at $295 \mathrm{~K}$ and on synchrotron data at 293 and $120 \mathrm{~K}$. Acta Cryst 1998;B54:687- 94

30. Ito $\mathrm{H}$, Iguchi $\mathrm{A}$, Hatano $\mathrm{T}$. Identification of urinary and intestinal bacterial metabolites of ellagitannin geraniin in rats. J Agric Food Chem 2008;56:393-400.

31. Ayaz F, Haylrlloglu AS, Alpay KS, Gruz J, Valentova' K, Ulrichova' J, et al. Phenolic acid contents of kale (Brassica oleraceae L. Var. Acephala DC.) extracts and their antioxidant and antimicrobial activities. Food Chem 2008;107:19-25.

32. Rahman AA, Moon SS. Antimicrobial phenolic derivatives from Dendranthema zawadskii var. Latilobum kitamura (Asteraceae). Arch Pharm Res 2007;30:1374-9.

33. Kelly EH, Anthony RT, Dennis JB. The B-ring hydroxyl flavonoid antioxidants: Chemistry, metabolism and structure-activity relationships. J Nutr Biochem 2002;13:572-84.

34. Kamsala RV, Lepakshi BMD, Padma Y, Venkata RR. Studies on antimicrobial and antioxidant properties of leaf extracts of Syzygium alternifolium. Int J Pharm Pharm Sci 2015;7:139-43.

35. Sekher Pannala A, Chan TS, O'Brien PJ, Rice-Evans CA. Flavonoid B-ring chemistry and antioxidant activity: Fast reaction kinetics. Biochem Biophys Res Commun 2001;282:1161-8.

36. Burda S, Oleszek W. Antioxidant and antiradical activities of flavonoids. J Agric Food Chem 2001:49:2774-9.

37. Hu JP, Calomme M, Lasure A, De Bruyne T, Pieters L, Vlietinck A, et al. Structure-activity relationship of flavonoids with superoxide scavenging activity. Biol Trace Elem Res 1995;47:327-31.

38. Van Acker SA, De Groot MJ, van den Berg DJ, Tromp MN, den Kelder GD, van der Vijgh WJ, et al. A quantum chemical explanation of the antioxidant activity of flavonoid. Chem Res Toxicol 1996;9:1305-12.

39. Arora A, Nair MG, Strasburg GM. Structure-activity relationships for antioxidant activities of a series of flavonoids in a liposomal system. Free Radic Biol Med 1998;24:1355-63. 